\title{
MULTIPLE-TERM APPROXIMATIONS FOR APPELL'S F FUNCTION
}

\author{
MAGDI A. EL-GABALI ${ }^{1}$
}

(Received 20 June 1995; revised 29 November 1995)

\begin{abstract}
In this paper computational issues of Appell's $F_{1}$ function$$
\mathrm{F}_{1}\left(\alpha, \beta, \beta^{\prime} ; \gamma ; x, y\right)=\sum_{m} \sum_{n} \frac{(\alpha)_{m+n}(\beta)_{m}\left(\beta^{\prime}\right)_{n}}{(\gamma)_{m+n} m ! n !} x^{m} y^{n}, \quad|x|<1,|y|<1
$$

are addressed. A novel technique is used in the derivation of highly efficient multiple-term approximations of this function (including asymptotic ones). Simple structured single- and double-term approximations, as the first two candidates of this multiple-term representation, are developed in closed form. Error analysis shows that the developed algorithms are superior to existing approximations for the same number of terms. The formulation presented is highly efficient and could be extended to a wide class of special functions.
\end{abstract}

\section{Introduction}

Hypergeometric functions in one and several variables have attracted the interest of many researchers over the years. This is attributed to the fact that most of the wellknown special functions can be represented in terms of these functions. Furthermore many problems in engineering and physical science are special cases of them, this is in addition to their applicability in other areas of analysis, statistics and operational research $[6-8,11-15]$.

Approximations of these functions are often required as a first step in the analysis of a given system where such functions appear. In this spirit, the author [3] derived a single-term approximation for the generalized elliptic-type integral

$$
\begin{gathered}
R_{\mu}(k, \alpha, \gamma)=\int_{0}^{\pi} \frac{\cos ^{2 \alpha-1}(\theta / 2) \sin ^{2 \gamma-2 \alpha-1}(\theta / 2)}{\left(1-k^{2} \cos \theta\right)^{\mu+1 / 2}} d \theta, \\
0 \leq k<1, \operatorname{Re}(\gamma)>\operatorname{Re}(\alpha)>0, \operatorname{Re}(\mu)>-0.5
\end{gathered}
$$

\footnotetext{
${ }^{1}$ Kuwait University, Department of Mathematics and Computer Science, P.O. Box 5969 Safat 13060 Kuwait

(C) Australian Mathematical Society, 1997, Serial-fee code 0334-2700/97
} 
in the form

$$
R_{\mu}(k, \alpha, \gamma) \approx \frac{B\left(\alpha, \gamma-\alpha-\zeta\left(\mu+\frac{1}{2}\right)\right)}{\left(1+k^{2}\right)^{\mu+1 / 2}} \quad \operatorname{Re}\left(\gamma-\alpha-\mu-\frac{1}{2}\right)>0,
$$

where $\zeta=2 k^{2} /\left(1+k^{2}\right)$.

This single term complements the range of parameters of another formula derived by Kalla, Leubner and Hubbell [10], viz.,

$$
\begin{gathered}
R_{\mu}(k, \alpha, \gamma) \approx \frac{B\left(\gamma-\alpha, \mu+\frac{1}{2}+\alpha-\gamma+\frac{\alpha-1}{\kappa}\right)}{\left(1-k^{2}\right)^{\mu+1 / 2} \kappa^{\gamma-\alpha}} \\
\operatorname{Re}\left(\mu+\frac{1}{2}+\alpha-\gamma\right)>0
\end{gathered}
$$

where $\kappa=2 k^{2} /\left(1-k^{2}\right)$. Galue, Kalla and Leubner [5] have derived a simple structured formula for the approximation of Hubbell rectangular source integral in the form

$$
h(a, b) \approx \frac{\sigma a}{2 \pi} \frac{b}{\left(2+a^{2}\right)} \frac{\Gamma\left(\frac{3}{2}\right) \Gamma\left(b^{2}+1\right)}{\Gamma\left(b^{2}+\frac{3}{2}\right)}, \quad \frac{a^{2} b^{2}}{2} \ll 1,
$$

where

$$
h(a, b)=\frac{\sigma}{4 \pi} \int_{0}^{b} \tan ^{-1}\left(\frac{a}{\sqrt{1+x^{2}}}\right) \frac{d x}{\sqrt{1+x^{2}}}, \quad a>0, b>0 .
$$

In the work done by Kalla and Galue [9], single-term approximations for some hypergeometric functions in one and two variables have been developed in some ranges of parameters and variables.

Accuracy requirements imposed by some applications require efficient simple structured approximations that may not be met with the single-term approximations. In this regard, the author has developed efficient multiple-term approximation formulas for $R_{\mu}(k, \alpha, \gamma)$ in the neighbourhood of $k^{2}=1$ [4]; which almost cover the entire range of parameters. It is evident that closed-form solutions reduce computations considerably and the improvement in accuracy by having $m$ terms instead of the single term is achieved by reduction of error from $O\left(h^{2}\right)$ to $O\left(h^{2 m}\right)$. The technique used may be interpreted as a rational approximation to a function that matches $m$ rational terms with $2 m$ terms in a Taylor series expansion, similar in nature to Pade's approximation.

In this paper, a novel technique is used in the development of efficient multiple-term approximations for Appell's $F_{1}$ function. The technique used is basically an extension and generalization of that used by the author in the development of the algorithms derived in [4] to hypergeometric functions in two variables. Single- and double-term approximations as the first two candidates of this representation are obtained in closed 
form. Computational analyses of the developed algorithms are compared with those approximations available in the literature.

Error analysis shows the effectiveness of the multiple-term approximations and the technique developed is superior to existing approximations for the same number of terms. The formulation presented here could be applied to a wide class of special functions.

The paper is organized as follows. Formulation of the multiple-term approximations for $|x| \ll 1,|y| \ll 1, x \rightarrow 1^{-}, y \rightarrow 1^{-}$and $x \rightarrow-\infty, y \rightarrow-\infty$ are presented in Section 2. Single- and double-term special cases are obtained in closed form and error measures are the subject of Section 3. Finally, a comparison analysis with the existing literature, using error measures, is given in Section 4.

\section{Multiple-term approximations for $\mathbf{F}_{1}\left(\alpha, \beta, \beta^{\prime} ; \gamma ; \mathbf{x}, \mathbf{y}\right)$}

In this section multiple-term approximations for Appell's $\mathrm{F}_{1}\left(\alpha, \beta, \beta^{\prime} ; \gamma ; x, y\right)$ function are derived. The series representation given by [1] and [2] is

$$
\mathrm{F}_{1}\left(\alpha, \beta, \beta^{\prime} ; \gamma ; x, y\right)=\sum_{m} \sum_{n} \frac{(\alpha)_{m+n}(\beta)_{m}\left(\beta^{\prime}\right)_{n}}{(\gamma)_{m+n} m ! n !} x^{m} y^{n}, \quad|x|<1,|y|<1 .
$$

The integral representation of this function is given by

$$
\begin{aligned}
\mathrm{F}_{1}\left(\alpha, \beta, \beta^{\prime} ; \gamma ; x, y\right)= & \frac{\Gamma(\alpha)}{\Gamma(\beta) \Gamma\left(\beta^{\prime}\right) \Gamma\left(\gamma-\beta-\beta^{\prime}\right)} \\
& \iint u^{\beta-1} v^{\beta^{\prime}-1}(1-u-v)^{\gamma-\beta-\beta^{\prime}}(1-u-v)^{-\alpha} d u d v,
\end{aligned}
$$

where $\operatorname{Re}(\beta)>0, \operatorname{Re}\left(\beta^{\prime}\right)>0, \operatorname{Re}\left(\gamma-\beta-\beta^{\prime}\right)>0$, and the domain in the $u v$ plane is the triangular area $u \geq 0, v \geq 0, u+v \leq 1$. Picard in [1] has pointed out that $\mathrm{F}_{1}\left(\alpha, \beta, \beta^{\prime} ; \gamma ; x, y\right)$ can be represented in a more useful way using a single integral in the form

$$
\begin{aligned}
& \mathrm{F}_{1}\left(\alpha, \beta, \beta^{\prime} ; \gamma ; x, y\right) \\
& \quad=\frac{1}{B(\alpha, \gamma-\alpha)} \int_{0}^{1} u^{\alpha-1}(1-u)^{\gamma-\alpha-1}(1-u x)^{-\beta}(1-u y)^{-\beta^{\prime}} d u .
\end{aligned}
$$

2.1. Multiple-term approximation for $F_{1}\left(\alpha, \beta, \beta^{\prime} ; \gamma ; x, y\right)$ for $|x| \ll 1,|y| \ll 1$. (i) It is not difficult to see that there exist complex values $A_{1}, A_{2}, \ldots, A_{m}$ and $z_{1}, z_{2}, \ldots, z_{m}[4]$ such that

$$
\sum_{j=1}^{m} A_{j}(1-u)^{-z_{j}} \approx(1-u x)^{-\beta}(1-u y)^{-\beta^{\prime}},
$$


where $m=1$ for a single term, $m=2$ for double term, ... etc. Upon using binomial expansions of both sides of (8) and equating equal powers of $u$ we arrive at

$$
\sum_{j=1}^{m} A_{j}\left(z_{j}\right)_{n}=\sum_{k=0}^{n}\left(\begin{array}{l}
n \\
k
\end{array}\right)\left(\beta^{\prime}\right)_{k}(\beta)_{n-k} x^{n-k} y^{k}
$$

The set of equations (9) can be written in the canonical form

$$
\sum_{j=1}^{m} A_{j} z_{j}^{n}=\phi_{n}(x, y), \quad n=0,1,2, \ldots, 2 m-1 .
$$

This represents a system of $2 m$ nonlinear equations in the unknowns $A_{1}, A_{2}, \ldots, A_{m}$ and $z_{1}, z_{2}, \ldots, z_{m}$. Solving (10), substituting the values of $A_{1}, A_{2}, \ldots, A_{m}$ and $z_{1}, z_{2}, \ldots, z_{m}$ into (8) and performing the integration given in (7) leads to a multipleterm approximation for Appell's $F_{1}$ function in the form

$$
\mathrm{F}_{1}\left(\alpha, \beta, \beta^{\prime} ; \gamma ; x, y\right) \approx \frac{1}{B(\alpha, \gamma-\alpha)} \sum_{j=1}^{m} A_{j} B\left(\alpha, \gamma-\alpha-z_{j}\right),
$$

where $\operatorname{Re}(\gamma)>\operatorname{Re}(\alpha)>\operatorname{Re}\left(z_{j}\right), \operatorname{Re}(\alpha)>0,|x| \ll 1,|y| \ll 1$.

2.2. Multiple-term approximation for $F_{1}\left(\alpha, \beta, \beta^{\prime} ; \gamma ; \mathbf{x}, \mathbf{y}\right)$ for $|\mathbf{x}| \ll 1,|y| \ll 1$ and $\operatorname{Re}(x)<0, \operatorname{Re}(y)<0$.

Replacing $u$ by $1-u$ in (7) results in

$$
\begin{aligned}
& \mathrm{F}_{1}\left(\alpha, \beta, \beta^{\prime} ; \gamma ; x, y\right) \\
& \quad=\frac{(1-x)^{-\beta}(1-y)^{-\beta^{\prime}}}{B(\alpha, \gamma-\alpha)} \int_{0}^{1} u^{\gamma-\alpha-1}(1-u)^{\alpha-1}\left(1-\frac{u x}{1-x}\right)^{-\beta}\left(1-\frac{u y}{1-y}\right)^{-\beta^{\prime}} d u .
\end{aligned}
$$

Let

$$
\hat{x}=\frac{x}{1-x}, \quad \hat{y}=\frac{y}{1-y} .
$$

(ii) There exist complex values $\hat{A}_{1}, \hat{A}_{2}, \ldots, \hat{A}_{m}$ and $\hat{z}_{1}, \hat{z}_{2}, \ldots, \hat{z}_{m}$ such that

$$
\sum_{j=1}^{m} \hat{A}_{j}(1-u)^{-\hat{z}_{j}} \approx(1-u \hat{x})^{-\beta}(1-u \hat{y})^{-\beta^{\prime}}
$$

Using binomial expansions of both sides of (14) and equating equal powers of $u$ results in

$$
\sum_{j=1}^{m} \hat{A}_{j}\left(-\hat{z}_{j}\right)_{n}=(-1)^{n} \sum_{k=0}^{n}\left(\begin{array}{l}
n \\
k
\end{array}\right)\left(\beta^{\prime}\right)_{k}(\beta)_{n-k} \hat{x}^{n-k} \hat{y}^{k},
$$


which can be written in the form

$$
\sum_{j=1}^{m} \hat{A}_{j} \hat{z}_{j}^{n}=\hat{\phi}_{n}(\hat{x}, \hat{y}), \quad n=0,1,2, \ldots, 2 m-1 .
$$

The multiple-term approximation for Appell's $F_{1}$ function is of the form

$$
\mathrm{F}_{1}\left(\alpha, \beta, \beta^{\prime} ; \gamma ; x, y\right) \approx \frac{(1-x)^{-\beta}(1-y)^{-\beta^{\prime}}}{B(\gamma-\alpha, \alpha)} \sum_{j=1}^{m} \hat{A}_{j} B\left(\gamma-\alpha, \alpha-\hat{z}_{j}\right),
$$

where $\operatorname{Re}(\gamma)>\operatorname{Re}(\alpha)>\operatorname{Re}\left(\hat{z}_{j}\right), \operatorname{Re}(\alpha)>0,|x| \ll 1,|y| \ll 1$.

2.3. Multiple-term approximation for $F_{1}\left(\alpha, \beta, \beta^{\prime} ; \gamma ; x, y\right)$ for $x \rightarrow 1^{-}$and $\mathbf{y} \rightarrow \mathbf{1}^{-}$.

With some simple transformations (7) can be written in the form

$$
\begin{aligned}
& \mathrm{F}_{1}\left(\alpha, \beta, \beta^{\prime} ; \gamma ; x, y\right) \\
& \quad=\frac{1}{B(\alpha, \gamma-\alpha)} \int_{0}^{\infty} \frac{\tau^{\alpha-1}}{(1+\tau)^{\gamma-\beta-\beta^{\prime}}}\left(1+\tau \delta_{x}\right)^{-\beta}\left(1+\tau \delta_{y}\right)^{-\beta^{\prime}} d \tau,
\end{aligned}
$$

$\delta_{x}=1-x, \delta_{y}=1-y,\left(\delta_{x}, \delta_{y}\right) \rightarrow\left(0^{+}, 0^{+}\right)$.

(i) There exist complex values $C_{1}, C_{2}, \ldots, C_{m}$ and $\omega_{1}, \omega_{2}, \ldots, \omega_{m}$ (cf. [4]) such that

$$
\sum_{j=1}^{m} C_{j}(1+\tau)^{-\omega_{j}} \approx\left(1+\delta_{x} \tau\right)^{-\beta}\left(1+\delta_{y} \tau\right)^{-\beta^{\prime}} .
$$

Performing binomial expansions on both sides of (19) and equating equal powers of $\tau$ results in

$$
\sum_{j=1}^{m} C_{j}\left(\omega_{j}\right)_{n}=\left(\begin{array}{l}
n \\
k
\end{array}\right)\left(\beta^{\prime}\right)_{k}(\beta)_{n-k} \delta_{x}^{n-k} \delta_{y}^{k}
$$

which can be written in the form

$$
\sum_{j=1}^{m} C_{j} \omega_{j}^{n}=\psi_{n}\left(\delta_{x}, \delta_{y}\right)=\phi_{n}\left(\delta_{x}, \delta_{y}\right), \quad n=0,1, \ldots, 2 m-1 .
$$

The multiple-term approximation for Appell's $F_{1}$ function is given by

$$
\mathrm{F}_{1}\left(\alpha, \beta, \beta^{\prime} ; \gamma ; x, y\right) \approx \frac{1}{B(\alpha, \gamma-\alpha)} \sum_{j=1}^{m} C_{j} B\left(\alpha, \gamma-\alpha-\beta-\beta^{\prime}+\omega_{j}\right),
$$

where $\operatorname{Re}(\gamma)>\operatorname{Re}(\alpha)>0, \operatorname{Re}\left(\gamma-\alpha-\beta-\beta^{\prime}+\omega_{j}\right)>0$, and $x \rightarrow 1^{-}, y \rightarrow 1^{-}$. 
2.4. Multiple-term approximation for $F_{1}\left(\alpha, \beta, \beta^{\prime} ; \gamma ; x, y\right) x \rightarrow-\infty$ and $y \rightarrow$ $-\infty$.

Using the substitution $\tau=1 / u-1$ in (7) results in

$$
\begin{aligned}
& \mathrm{F}_{1}\left(\alpha, \beta, \beta^{\prime} ; \gamma ; x, y\right)=\frac{(1-x)^{\beta}(1-y)^{\beta^{\prime}}}{B(\gamma-\alpha, \alpha)} \times \\
& \quad \int_{0}^{\infty} \frac{\tau^{\gamma-\alpha-1}}{(1+\tau) \gamma-\beta-\beta^{\prime}}\left(1+\frac{\tau}{1-x}\right)^{-\beta}\left(1+\frac{\tau}{1-y}\right)^{-\beta^{\prime}} d \tau .
\end{aligned}
$$

Let $\varepsilon_{x}=\frac{1}{1-x}, \varepsilon_{y}=\frac{1}{1-y},\left(\varepsilon_{x}, \varepsilon_{y}\right) \rightarrow\left(0^{+}, 0^{+}\right)$.

(i) There exist complex values $E_{1}, E_{2}, \ldots, E_{m}$ and $\lambda_{1}, \lambda_{2}, \ldots, \lambda_{m}$ such that

$$
\sum_{j=1}^{m} E_{j}(1+\tau)^{-\lambda_{j} \approx}\left(1+\varepsilon_{x} \tau\right)^{-\beta}\left(1+\varepsilon_{y} \tau\right)^{-\beta^{\prime}} .
$$

Performing binomial expansions on both sides of (24) and equating equal powers of $\tau$ results in

$$
\sum_{j=1}^{m} E_{j}\left(\lambda_{j}\right)_{n}=\left(\begin{array}{l}
n \\
k
\end{array}\right)\left(\beta^{\prime}\right)_{k}(\beta)_{n-k} \varepsilon_{x}^{n-k} \varepsilon_{y}^{k}
$$

which can be written in the form

$$
\sum_{j=1}^{m} E_{j} \lambda_{j}^{n}=\theta_{n}\left(\varepsilon_{x}, \varepsilon_{y}\right), \quad n=0,1,2 \ldots, 2 m-1 .
$$

The multiple-term approximation for Appell's $F_{1}$ function is given by

$$
\begin{aligned}
& \mathrm{F}_{1}\left(\alpha, \beta, \beta^{\prime} ; \gamma ; x, y\right) \approx \frac{(1-x)^{-\beta}(1-y)^{-\beta^{\prime}}}{B(\gamma-\alpha, \alpha)} \sum_{j=1}^{m} E_{j} B\left(\gamma-\alpha, \alpha-\beta-\beta^{\prime}+\lambda_{j}\right), \\
& \operatorname{Re}(\gamma)>\operatorname{Re}(\alpha)>0, \operatorname{Re}\left(\alpha-\beta-\beta^{\prime}+\lambda_{j}\right)>0, x \rightarrow-\infty \text { and } y \rightarrow-\infty
\end{aligned}
$$

Asymptotic expansion formula for $\mathrm{F}_{1}\left(\alpha, \beta, \beta^{\prime} ; \gamma ; x, y\right) x \rightarrow-\infty$ and $y \rightarrow-\infty$.

By applying a binomial expansion to the relevant terms in (23), we arrive at

$$
\begin{aligned}
& \mathrm{F}_{1}\left(\alpha, \beta, \beta^{\prime} ; \gamma ; x, y\right) \approx \frac{(1-x)^{\beta}(1-y)^{\beta^{\prime}}}{B(\gamma-\alpha, \alpha)} \times \\
& \quad \sum_{n=1}^{N} \frac{(-1)^{n} B\left(\gamma-\alpha+n, \alpha-\beta-\beta^{\prime}-n\right)}{n !} \sum_{k=0}^{n}\left(\begin{array}{l}
n \\
k
\end{array}\right)\left(\beta^{\prime}\right)_{k}(\beta)_{n-k} \varepsilon_{x}^{n-k} \varepsilon_{y}^{k}
\end{aligned}
$$

for $x \rightarrow-\infty$ and $y \rightarrow-\infty$ and where $N$ can be as large as is compatible with $\operatorname{Re}\left(\alpha-\beta-\beta^{\prime}-n\right)>0, n=0,1, \ldots, N$. 
3. Single- and double-term approximations for $F_{1}\left(\alpha, \beta, \beta^{\prime} ; \gamma ; x, y\right)$

\subsection{Single-term approximations}

Case 1a. Single-term approximation for $\mathrm{F}_{1}\left(\alpha, \beta, \beta^{\prime} ; \gamma ; x, y\right)$ for $|x| \ll 1,|y| \ll 1$.

Setting $m=1$ in (9) and $n=0,1$ results in $A_{1}=1, z_{1}=\beta x+\beta^{\prime} y$. Substituting these values in (22), the single-term approximation is found as

$$
\mathrm{F}_{1}\left(\alpha, \beta, \beta^{\prime} ; \gamma ; x, y\right) \approx \frac{B\left(\alpha, \gamma-\alpha-\beta x-\beta^{\prime} y\right)}{B(\alpha, \gamma-\alpha)}
$$

$\operatorname{Re}(\gamma)>\operatorname{Re}(\alpha)>\operatorname{Re}\left(z_{1}\right), \operatorname{Re}(\alpha)>0,|x| \ll 1,|y| \ll 1$.

Case1b. Single-term approximation for $\mathrm{F}_{1}\left(\alpha, \beta, \beta^{\prime} ; \gamma ; x, y\right)$ for $|x| \ll 1,|y| \ll 1$ and $\operatorname{Re}(x)<0, \operatorname{Re}(y)<0$.

Setting $m=1$ and $n=0,1$ in (15) results in $\hat{A}_{1}=1, \hat{z}_{1}=-\left(\beta \hat{x}+\beta^{\prime} \hat{y}\right)$. Substitution of these values in (17) gives the single-term approximation

$$
\mathrm{F}_{1}\left(\alpha, \beta, \beta^{\prime} ; \gamma ; x, y\right) \approx \frac{(1-x)^{-\beta}(1-y)^{-\beta^{\prime}}}{B(\gamma-\alpha, \alpha)} B\left(\gamma-\alpha, \alpha+\beta \hat{x}+\beta^{\prime} \hat{y}\right),
$$

where $\operatorname{Re}(\gamma)>\operatorname{Re}(\alpha)>0, \operatorname{Re}\left(\alpha+\beta \hat{x}+\beta^{\prime} \hat{y}\right)>0$.

REMARKS. (1) Formulas (29) and (30) are identical to equations [9, (24), (15)] developed Kalla and Galue.

(2) The error in the computation of $\mathrm{F}_{1}\left(\alpha, \beta, \beta^{\prime} ; \gamma ; x, y\right)$ using (29) and (30) is of the order of $O\left(h^{2}\right)$, where $h=\max (|x|,|y|)$ in (29) and $h=\max (\mid x /(1-$ $x) \mid),|y /(1-y)|)$ in $(30)$.

Case 2. Single-term approximation for $\mathrm{F}_{1}\left(\alpha, \beta, \beta^{\prime} ; \gamma ; x, y\right), x \rightarrow 1^{-}$and $y \rightarrow 1^{-}$.

Setting $m=1$ and $n=0,1$ in (20) results in $C_{1}=1, \omega_{1}=\beta \delta_{x}+\beta^{\prime} \delta_{y}$. Substituting these values in (22), we obtain the single-term approximation

$$
\mathrm{F}_{1}\left(\alpha, \beta, \beta^{\prime} ; \gamma ; x, y\right) \approx \frac{B\left(\alpha, \gamma-\alpha-\beta x-\beta^{\prime} y\right)}{B(\alpha, \gamma-\alpha)},
$$

where $\operatorname{Re}(\gamma)>\operatorname{Re}(\alpha)>0, \operatorname{Re}\left(\gamma-\alpha-\beta x-\beta^{\prime} y\right)>0, x \rightarrow 1^{-}, y \rightarrow 1^{-}$.

Case 3. Single-term approximation for $\mathrm{F}_{1}\left(\alpha, \beta, \beta^{\prime} ; \gamma ; x, y\right), x \rightarrow-\infty$ and $y \rightarrow$ $-\infty$. 
Setting $m=1$ and $n=0,1$ in (25) results in $E_{1}=1, \lambda_{1}=\left(\beta \varepsilon_{x}+\beta^{\prime} \varepsilon_{y}\right)$. Substituting these values in (27) we arrive at the single-term approximation

$$
\begin{aligned}
& \mathrm{F}_{1}\left(\alpha, \beta, \beta^{\prime} ; \gamma ; x, y\right) \approx \\
& \quad \frac{(1-x)^{-\beta}(1-y)^{-\beta^{\prime}}}{B(\gamma-\alpha, \alpha)} B\left\{\left(\gamma-\alpha, \alpha+\beta\left(\frac{x}{1-x}\right)+\beta^{\prime}\left(\frac{y}{1-y}\right)\right\},\right.
\end{aligned}
$$

where $\operatorname{Re}(\gamma)>\operatorname{Re}(\alpha)>0, \operatorname{Re}\left(\alpha+\beta\left(\frac{x}{1-x}\right)+\beta^{\prime}\left(\frac{y}{1-y}\right)\right)>0$.

\subsection{Double-term approximations}

Case 1a. Two-term approximations for $\mathrm{F}_{1}\left(\alpha, \beta, \beta^{\prime} ; \gamma ; x, y\right)$ for $|x| \ll 1,|y| \ll 1$.

Setting $m=2$ and $n=0,1,2,3$ in (9) results in

$$
A_{1}\left(z_{1}\right)_{n}+A_{2}\left(z_{2}\right)_{n}=\sum_{k=0}^{n}\left(\begin{array}{l}
n \\
k
\end{array}\right)\left(\beta^{\prime}\right)_{k}(\beta)_{n-k} x^{n-k} y^{k}, \quad n=0,1,2,3 .
$$

The four nonlinear equations can be written in the form

$$
A_{1} z_{1}^{n}+A_{2} z_{2}^{n}=\phi_{n}(x, y), \quad n=0,1,2,3,
$$

where

$$
\begin{aligned}
\phi_{0}(x, y) & =1, \\
\phi_{1}(x, y) & =\beta x+\beta^{\prime} y, \\
\phi_{2}(x, y) & =\phi_{1}^{2}(x, y)-\phi_{1}(x, y)+\beta x^{2}+\beta^{\prime} y^{2} \\
\phi_{3}(x, y) & =-2 \phi_{1}(x, y)-3 \phi_{2}(x, y)+\phi_{1}^{3}(x, y)+\beta(3 \beta+2) x^{3} \\
& \quad+\beta^{\prime}\left(3 \beta^{\prime}+2\right) y^{3}+3 \beta \beta^{\prime} x y(x+y) .
\end{aligned}
$$

(i) Approximate two-term solution

Setting $A_{1}=A_{2}$ and solving the first three equations of (34) results in a solution of the form

$$
\mathrm{F}_{1}\left(\alpha, \beta, \beta^{\prime} ; \gamma ; x, y\right) \approx \frac{1}{2 B(\alpha, \gamma-\alpha)}\left\{B\left(\alpha, \gamma-\alpha-z_{1}\right)+B\left(\alpha, \gamma-\alpha-z_{2}\right)\right\}
$$

with $\operatorname{Re}(\gamma)>\operatorname{Re}(\alpha)>0, \operatorname{Re}\left(\gamma-\alpha-z_{1}\right)>0, \operatorname{Re}\left(\gamma-\alpha-z_{2}\right)>0$, where

$$
\begin{aligned}
& z_{1}=\beta x+\beta^{\prime} y+i \sqrt{\beta x(1-x)+\beta^{\prime} y(1-y)}, \\
& z_{2}=\beta x+\beta^{\prime} y-i \sqrt{\beta x(1-x)+\beta^{\prime} y(1-y)} .
\end{aligned}
$$

Error estimate: let $h=\max (|x|,|y|)$. The error in the computation of $\mathrm{F}_{1}\left(\alpha, \beta, \beta^{\prime}\right.$; $\gamma ; x, y)$ using (36) is $O\left(h^{3}\right)$. 
(ii) A highly efficient two-term approximation for $\mathrm{F}_{1}\left(\alpha, \beta, \beta^{\prime} ; \gamma ; x, y\right)$

The four nonlinear equations of (34) are solved for $\left(A_{1}, A_{2}\right)$ and $\left(z_{1}, z_{2}\right)$ and the first two equations of (34) are solved for $A_{1}$ and $A_{2}$, leading to

$$
A_{1}=\frac{\phi_{1}-z_{2}}{z_{1}-z_{2}}, \quad A_{2}=\frac{z_{1}-\phi_{1}}{z_{1}-z_{2}} .
$$

Substitution of (38) in the last two equations of (34) leads to two equations in $z_{1}$ and $z_{2}$ which can be linearized by making the change of variables

$$
\mu=\frac{z_{1}+z_{2}}{2}, \quad v=z_{1} z_{2} .
$$

The solution for $\mu$ and $v$ is given by

$$
\mu=\frac{\phi_{3}-\phi_{1} \phi_{2}}{2\left(\phi_{2}-\phi_{1}^{2}\right)}, \quad \nu=\frac{\phi_{1} \phi_{3}-\phi_{2}^{2}}{\phi_{2}-\phi_{1}^{2}} .
$$

The solution of (34) results in

$$
z_{1}=\mu+\sqrt{\mu^{2}-v}, \quad z_{2}=\mu-\sqrt{\mu^{2}-v}
$$

and a highly efficient two-term approximation for $\mathrm{F}_{1}\left(\alpha, \beta, \beta^{\prime} ; \gamma ; x, y\right)$ is given by

$$
\begin{aligned}
& \mathrm{F}_{1}\left(\alpha, \beta, \beta^{\prime} ; \gamma ; x, y\right) \\
& \quad \approx \frac{1}{B(\alpha, \gamma-\alpha)}\left\{A_{1} B\left(\alpha, \gamma-\alpha-z_{1}\right)+A_{2} B\left(\alpha, \gamma-\alpha-z_{2}\right)\right\}
\end{aligned}
$$

with $\operatorname{Re}(\gamma)>\operatorname{Re}(\alpha)>0, \operatorname{Re}\left(\gamma-\alpha-z_{1}\right)>0, \operatorname{Re}\left(\gamma-\alpha-z_{2}\right)>0$, and $|x| \ll$ $1,|y| \ll 1$. It should be noted that the error in the computation of $\mathrm{F}_{1}\left(\alpha, \beta, \beta^{\prime} ; \gamma ; x, y\right)$ using (42) is $O\left(h^{4}\right)$.

Case 1b. Double-term approximations for $\mathrm{F}_{1}\left(\alpha, \beta, \beta^{\prime} ; \gamma ; x, y\right),|x| \ll 1,|y| \ll 1$ and $\operatorname{Re}(x)<0, \operatorname{Re}(y)<0$.

Setting $m=2$ in (16) results in

$$
\hat{A}_{1} \hat{z}_{1}^{n}+\hat{A}_{2} \hat{z}_{2}^{n}=\hat{\phi}_{n}(\hat{x}, \hat{y})=\phi_{n}(-\hat{x},-\hat{y})=\rho_{n}(\hat{x}, \hat{y}), n=0,1, \ldots, 2 m-1,(43)
$$

where

$$
\begin{aligned}
\rho_{0}(\hat{x}, \hat{y})= & 1, \quad \rho_{1}(\hat{x}, \hat{y})=-\beta \hat{x}-\beta^{\prime} \hat{y}, \\
\rho_{2}(\hat{x}, \hat{y})= & \rho_{1}^{2}(\hat{x}, \hat{y})+\rho_{1}(\hat{x}, \hat{y})+\beta \hat{x}^{2}+\beta^{\prime} \hat{y}^{2}, \\
\rho_{3}(\hat{x}, \hat{y})= & 2 \rho_{1}(\hat{x}, \hat{y})-3 \rho_{2}(\hat{x}, \hat{y})+\rho_{1}^{3}(\hat{x}, \hat{y})-\beta(3 \beta+2) \hat{x}^{3} \\
& -\beta^{\prime}\left(3 \beta^{\prime}+2\right) \hat{y}^{3}-3 \beta \beta^{\prime} \hat{x} \hat{y}(\hat{x}+\hat{y}) .
\end{aligned}
$$


(i) Approximate two-term solution

Setting $\hat{A}_{1}=\hat{A}_{2}$ and solving the first three equations of (43) results in the approximation

$$
\begin{aligned}
& \mathrm{F}_{1}\left(\alpha, \beta, \beta^{\prime} ; \gamma ; x, y\right) \\
& \quad \approx \frac{(1-x)^{-\beta}(1-y)^{-\beta^{\prime}}}{2 B(\gamma-\alpha, \alpha)}\left\{B\left(\gamma-\alpha, \alpha-\hat{z}_{1}\right)+B\left(\gamma-\alpha, \alpha-\hat{z}_{2}\right)\right\},
\end{aligned}
$$

where

$$
\begin{aligned}
& \hat{z}_{1}=-\left(\beta \frac{x}{1-x}+\beta^{\prime} \frac{y}{1-y}\right)+i \sqrt{\frac{-\beta x}{(1-x)^{2}}+\frac{-\beta^{\prime} y}{(1-y)^{2}}}, \\
& \hat{z}_{2}=-\left(\beta \frac{x}{1-x}+\beta^{\prime} \frac{y}{1-y}\right)-i \sqrt{\frac{-\beta x}{(1-x)^{2}}+\frac{-\beta^{\prime} y}{(1-y)^{2}}} .
\end{aligned}
$$

Error estimates: let $h=\max (|x /(1-x)|,|y /(1-y)|)$. The error in the computation of $\mathrm{F}_{1}\left(\alpha, \beta, \beta^{\prime} ; \gamma ; x, y\right)$ using (45) is $O\left(h^{3}\right)$.

(ii) A highly efficient two-term approximation for $\mathrm{F}_{1}\left(\alpha, \beta, \beta^{\prime} ; \gamma ; x, y\right)$

This case is similar to that used in deriving (42). The two-term approximation is given by

$$
\begin{aligned}
& \mathrm{F}_{1}\left(\alpha, \beta, \beta^{\prime} ; \gamma ; x, y\right) \\
& \quad \approx \frac{(1-x)^{-\beta}\left((1-y)^{-\beta^{\prime}}\right.}{B(\gamma-\alpha, \alpha)}\left\{\hat { A } _ { 1 } B \left(\left(\gamma-\alpha, \alpha-\hat{z}_{1}\right)+\hat{A}_{2} B\left(\left(\gamma-\alpha, \alpha-\hat{z}_{2}\right)\right\},\right.\right.
\end{aligned}
$$

where

$$
\begin{aligned}
\hat{A}_{1}=\frac{\rho_{1}-\hat{z}_{2}}{\hat{z}_{1}-\hat{z}_{2}}, & \hat{A}_{2}=\frac{\hat{z}_{1}-\rho_{1}}{\hat{z}_{1}-\hat{z}_{2}}, \\
\hat{z}_{1}=\mu+\sqrt{\mu^{2}-v}, & \hat{z}_{2}=\mu-\sqrt{\mu^{2}-v}
\end{aligned}
$$

and $\mu$ and $\nu$ are given by

$$
\mu=\frac{\rho_{3}-\rho_{1} \rho_{2}}{2\left(\rho_{2}-\rho_{1}^{2}\right)}, \quad v=\frac{\rho_{1} \rho_{3}-\rho_{2}^{2}}{\rho_{2}-\rho_{1}^{2}} .
$$

The error in the computation of $\mathrm{F}_{1}\left(\alpha, \beta, \beta^{\prime} ; \gamma ; x, y\right)$ is $O\left(h^{4}\right)$.

Case 2. Two-term approximation for $\mathrm{F}_{1}\left(\alpha, \beta, \beta^{\prime} ; \gamma ; x, y\right), x \rightarrow 1^{-}, y \rightarrow 1^{-}$.

Setting $m=2$ and $n=0,1,2,3$ in (20) leads to

$$
C_{1}\left(\omega_{1}\right)_{n}+C_{2}\left(\omega_{2}\right)_{n}=\sum_{k=0}^{n}\left(\begin{array}{l}
n \\
k
\end{array}\right)\left(\beta^{\prime}\right)_{k}(\beta)_{n-k} \delta_{x}^{n-k} \delta_{y}^{k}, \quad n=0,1,2,3
$$


The four nonlinear equations can be written in the form

$$
C_{1} \omega_{1}^{n}+C_{2} \omega_{2}^{n}=\psi_{n}\left(\delta_{x}, \delta_{y}\right)=\phi_{n}\left(\delta_{x}, \delta_{y}\right), \quad n=0,1,2,3 .
$$

This case is similar to case $1 \mathrm{a}$ above and the values of $\phi_{n}\left(\delta_{x}, \delta_{y}\right)(n=0,1,2,3)$ are given in (35).

(i) Approximate two-term solution for $\mathrm{F}_{1}\left(\alpha, \beta, \beta^{\prime} ; \gamma ; x, y\right)$.

The solution is obtained by a method similar to that of case $1 \mathrm{a}$ and is given by

$$
\begin{aligned}
\mathrm{F}_{1}\left(\alpha, \beta, \beta^{\prime} ; \gamma ; x, y\right) \approx \frac{1}{2 B(\alpha, \gamma-\alpha)} \times \\
\left\{B\left(\alpha, \gamma-\alpha-\beta-\beta^{\prime}+\omega_{1}\right)+B\left(\alpha, \gamma-\alpha-\beta-\beta^{\prime}+\omega_{2}\right)\right\},
\end{aligned}
$$

where

$$
\begin{aligned}
& \omega_{1}=\beta x+\beta^{\prime} y+i \sqrt{\beta x(1-x)+\beta^{\prime} y(1-y)}, \\
& \omega_{2}=\beta x+\beta^{\prime} y-i \sqrt{\beta x(1-x)+\beta^{\prime} y(1-y)} .
\end{aligned}
$$

Error estimate: let $h=\max (1-x, 1-y)$. The error in the computation of $\mathrm{F}_{1}\left(\alpha, \beta, \beta^{\prime} ; \gamma ; x, y\right)$ is $O\left(h^{3}\right)$.

(ii) Highly efficient two-term approximation for $\mathrm{F}_{1}\left(\alpha, \beta, \beta^{\prime} ; \gamma ; x, y\right), x \rightarrow 1^{-}$and $y \rightarrow 1^{-}$.

This method is similar to case $1 \mathrm{a}$ and the solution is given by

$$
\begin{aligned}
& \mathrm{F}_{1}\left(\alpha, \beta, \beta^{\prime} ; \gamma ; x, y\right) \approx \frac{1}{B(\alpha, \gamma-\alpha)} \times \\
& \quad\left\{C_{1} B\left(\alpha, \gamma-\alpha-\beta-\beta^{\prime}+\omega_{1}\right)+C_{2} B\left(\alpha, \gamma-\alpha-\beta-\beta^{\prime}+\omega_{2}\right)\right\},
\end{aligned}
$$

where

$$
\begin{array}{cc}
C_{1}=\frac{\phi_{1}\left(\delta_{x}, \delta_{y}\right)-\omega_{2}}{\omega_{1}-\omega_{2}}, & C_{2}=\frac{\omega_{1}-\phi_{1}\left(\delta_{x}, \delta_{y}\right)}{\omega_{1}-\omega_{2}}, \\
\omega_{1}=\mu+\sqrt{\mu^{2}-\nu}, & \omega_{2}=\mu-\sqrt{\mu^{2}-v},
\end{array}
$$

$\mu$ and $v$ are given by

$$
\begin{gathered}
\mu=\frac{\phi_{3}\left(\delta_{x}, \delta_{y}\right)-\phi_{1}\left(\delta_{x}, \delta_{y}\right) \phi_{2}\left(\delta_{x}, \delta_{y}\right)}{2\left(\phi_{2}\left(\delta_{x}, \delta_{y}\right)-\phi_{1}^{2}\left(\delta_{x}, \delta_{y}\right)\right)}, \\
v=\frac{\phi_{1}\left(\delta_{x}, \delta_{y}\right) \phi_{3}\left(\delta_{x}, \delta_{y}\right)-\phi_{2}^{2}\left(\delta_{x}, \delta_{y}\right)}{\phi_{2}\left(\delta_{x}, \delta_{y}\right)-\phi_{1}^{2}\left(\delta_{x}, \delta_{y}\right)},
\end{gathered}
$$

and the set of values of $\phi_{n}\left(\delta_{x}, \delta_{y}\right),(n=0,1,2,3)$ is given by (35).

Error estimate: the error in the computation of (55) is $O\left(h^{4}\right)$. 
Case 3. Two-term approximation for $\mathrm{F}_{1}\left(\alpha, \beta, \beta^{\prime} ; \gamma ; x, y\right), x \rightarrow-\infty$ and $y \rightarrow-\infty$. Setting $m=2$ and $n=0,1,2,3$ in (26) results in

$$
E_{1} \lambda_{1}^{n}+E_{2} \lambda_{2}^{n}=\phi_{n}\left(\varepsilon_{x}, \varepsilon_{y}\right), \quad n=0,1,2,3,
$$

where $\phi_{n}\left(\varepsilon_{x}, \varepsilon_{y}\right)(n=0,1,2,3)$ is given by (35).

(i) Approximate two-term solution

Setting $E_{1}=E_{2}$ and solving the first three equations of (59) results in the solution

$$
\begin{aligned}
\mathrm{F}_{1}\left(\alpha, \beta, \beta^{\prime} ; \gamma ; x, y\right) \approx \frac{(1-x)^{-\beta}(1-y)^{-\beta^{\prime}}}{2 B(\gamma-\alpha, \alpha)} \times \\
\left\{B\left(\gamma-\alpha, \alpha-\beta-\beta^{\prime}+\lambda_{1}\right)+B\left(\gamma-\alpha, \alpha-\beta-\beta^{\prime}+\lambda_{2}\right)\right\},
\end{aligned}
$$

where

$$
\begin{aligned}
& \lambda_{1}=\frac{\beta}{1-x}+\frac{\beta^{\prime}}{1-y}+i \sqrt{\frac{-\beta x}{(1-x)^{2}}+\frac{-\beta^{\prime} x}{(1-x)^{2}}}, \\
& \lambda_{2}=\frac{\beta}{1-x}+\frac{\beta^{\prime}}{1-y}-i \sqrt{\frac{-\beta x}{(1-x)^{2}}+\frac{-\beta^{\prime} x}{(1-x)^{2}}} .
\end{aligned}
$$

Error estimate: let $h=\max (1 /(1-x), 1 /(1-y))$. The error in the computation of $\mathrm{F}_{1}\left(\alpha, \beta, \beta^{\prime} ; \gamma ; x, y\right)$ using $(60)$ is $O\left(h^{3}\right)$.

(ii) A highly efficient two-term approximation for $\mathrm{F}_{1}\left(\alpha, \beta, \beta^{\prime} ; \gamma ; x, y\right) x \rightarrow-\infty$ and $y \rightarrow-\infty$

The solution is given by

$$
\begin{aligned}
& \mathrm{F}_{1}\left(\alpha, \beta, \beta^{\prime} ; \gamma ; x, y\right) \approx \frac{(1-x)^{-\beta}(1-y)^{-\beta^{\prime}}}{B(\gamma-\alpha, \alpha)} \times \\
&\left\{E_{1} B\left(\gamma-\alpha, \alpha-\beta-\beta^{\prime}+\lambda_{1}\right)+E_{2} B\left(\gamma-\alpha, \alpha-\beta-\beta^{\prime}+\lambda_{2}\right)\right\},
\end{aligned}
$$

where

$$
\begin{array}{cc}
E_{1}=\frac{\phi_{1}\left(\varepsilon_{x}, \varepsilon_{y}\right)-\lambda_{2}}{\lambda_{1}-\lambda_{2}}, & E_{2}=\frac{\lambda_{1}-\phi_{1}\left(\varepsilon_{x}, \varepsilon_{y}\right)}{\lambda_{1}-\lambda_{2}}, \\
\lambda_{1}=\mu+\sqrt{\mu^{2}-\nu}, & \lambda_{2}=\mu-\sqrt{\mu^{2}-\nu},
\end{array}
$$

and $\mu$ and $v$ are given by

$$
\begin{gathered}
\mu=\frac{\phi_{3}\left(\varepsilon_{x}, \varepsilon_{y}\right)-\phi_{1}\left(\varepsilon_{x}, \varepsilon_{y}\right) \phi_{2}\left(\varepsilon_{x}, \varepsilon_{y}\right)}{2\left(\phi_{2}\left(\varepsilon_{x}, \varepsilon_{y}\right)-\phi_{1}^{2}\left(\varepsilon_{x}, \varepsilon_{y}\right)\right)}, \\
\nu=\frac{\phi_{1}\left(\varepsilon_{x}, \varepsilon_{y}\right) \phi_{3}\left(\varepsilon_{x}, \varepsilon_{y}\right)-\phi_{2}^{2}\left(\varepsilon_{x}, \varepsilon_{y}\right)}{\phi_{2}\left(\varepsilon_{x}, \varepsilon_{y}\right)-\phi_{1}^{2}\left(\varepsilon_{x}, \varepsilon_{y}\right)} .
\end{gathered}
$$

Error estimates: for $h=\max (1 /(1-x), 1 /(1-y))$ the error in the computation of $\mathrm{F}_{1}\left(\alpha, \beta, \beta^{\prime} ; \gamma ; x, y\right)$ using $(62)$ is $O\left(h^{4}\right)$. 


\section{Error analysis and conclusion}

In this section, errors measures, comparison analysis and conclusions are presented. The approximation formulas for $\mathrm{F}_{1}\left(\alpha, \beta, \beta^{\prime} ; \gamma ; x, y\right)$ have been developed in terms of the beta function. Since the arguments of this function should have a positive real part, different formulas have been derived in the case of two-term approximations to meet this requirement for different ranges of parameters. An account of error measures as given in Section 3 can be summarized as follows.

Error estimates:

(i) The error in computing $\mathrm{F}_{1}\left(\alpha, \beta, \beta^{\prime} ; \gamma ; x, y\right)$ using (29) - (32) is $O\left(h^{2}\right)$;

(ii) the error in computing $\mathrm{F}_{1}\left(\alpha, \beta, \beta^{\prime} ; \gamma ; x, y\right)$ using (36), (45), (53) and (60) is $O\left(h^{3}\right)$;

(iii) the error in computing $\mathrm{F}_{1}\left(\alpha, \beta, \beta^{\prime} ; \gamma ; x, y\right)$ using (42), (47), (55) and (62) is $O\left(h^{4}\right)$.

It is not difficult to see that $m$ terms of the most efficient multiple-term approximations for $\mathrm{F}_{1}\left(\alpha, \beta, \beta^{\prime} ; \gamma ; x, y\right)$ are equivalent to $m(2 m+1)$ terms of the series expansion (5) or the asymptotic expansion (28) to achieve the same order of error. For example, two terms of the most efficient approximation (42) are equivalent to 10 terms of the series expansion of (5) to achieve an error $O\left(h^{4}\right)$. This analysis shows how efficient the multiple-term approximations are when compared to existing literature. It should also be noted that the approximation formulas developed in [9] for the approximations of the $F_{1}\left(\alpha, \beta, \beta^{\prime} ; \gamma ; x, y\right)$ function are simply special cases of our general formulation for case 1 and for $m=1$. However, the algorithms developed in the present paper support not only different ranges of variables (including asymptotic behaviour) but also give more flexibility by controlling the resolution through the selection of $m$. Thus, in conclusion, the algorithms developed in this paper are extremely useful in a large number of applications in which approximations of Appell's $\mathrm{F}_{1}\left(\alpha, \beta, \beta^{\prime} ; \gamma ; x, y\right)$ function are required.

\section{Acknowledgement}

This research was supported by grant SM 122 of Kuwait University.

\section{References}

[1] A. Erdelyi, Higher transcendental functions, Volume 1 (McGraw-Hill, New York, 1953).

[2] H. Exton, Multiple hypergeometric functions and applications (Wiley \& Sons, New York, 1976). 
[3] M. El-Gabali, "Single term asymptotic approximations for the generalized elliptic-type integrals", J. Revista de la Academia de Ciencias 6 (1994) 105-118.

[4] M. El-Gabali, "Efficient multiple term approximations for the generalized elliptic-type integrals", J. Austral. Math. Soc. Ser. B 38 (1997) 411-426.

[5] L. Galue, S. Kalla and C. Leubner, "Algorithms for the approximation of the generalized Hubbell rectangular source integrals”, Radiat. Phys. Chem. 43 (1994) 497-502.

[6] L. Galue and V. Kiryakova, "Further results on a family of generalized radiation integrals", Radiat. Phys. Chem. 43 (1994) 573-579.

[7] M. L. Glasser, "Solution of the problem 83 - 6 by J. H. Hubbell", SIAM Rev. 26 (1984) 276-278.

[8] S. L. Kalla, "The Hubbell rectangular source integral and its generalizations", Radiat. Phys. Chem. 41 (1993) 775-781.

[9] S. L. Kalla and L. Galue, "Simple algorithms for the approximation of the hypergeometric functions", J. Alg. Groups and Geom. 11 (1994) 347-359.

[10] S. L. Kalla, C. Leubner and J. H. Hubbell, "Further results on generalized elliptic-type integrals", Appl. Anal. 25 (1987) 269-274.

[11] S. L. Kalla, B. Al-Sagabi and S. Conde, "Some results related to radiation field problem", Hadronic Journal 10 (1987) 221-230.

[12] N. N. Lebedev, Special functions and their applications (Dover Publications, New York, 1972).

[13] A. M. Mathai and R. A. Saxena, Generalized hypergeometric functions with applications in Statistics and Physical Sciences, Volume 348 of Lecture notes in mathematics (Springer-Verlag, Berlin, 1973).

[14] J. Seaborn, Hypergeometric functions and their applications, Volume 1 (Springer-Verlag, New York, 1991).

[15] D. M. Timus, "Some further results on the Hubbell rectangular source integral", J. Comput. Appl. Math. 45 (1993) 331-338. 